\title{
A SURVEY ON QUESTION ANSWERING SYSTEMS: THE ADVANCES OF FUZZY LOGIC
}

\author{
Eman Mohamed Nabil Alkholy ${ }^{1}$, Mohamed Hassan Haggag ${ }^{1}$ and \\ Constantine's Koutsojannis ${ }^{2}$ \\ ${ }^{1}$ Faculty of Computers \& Information, Helwan University, Cairo, Egypt \\ ${ }^{2}$ Health Physics \& Computational Intelligence Lab, TEI of Western Greece, \\ Aigion
}

\begin{abstract}
In real world computing environment with using a computer to answer questions has been a human dream since the beginning of the digital era, Question-answering systems are referred to as intelligent systems, that can be used to provide responses for the questions being asked by the user based on certain facts or rules stored in the knowledge base it can generate answers of questions asked in natural, and the first main idea of fuzzy logic was to working on the problem of computer understanding of natural language, so this survey paper provides an overview on what Question-Answering is and its system architecture and the possible relationship and different with fuzzy logic, as well as the previous related research with respect to approaches that were followed. At the end, the survey provides an analytical discussion of the proposed QA models, along or combined with fuzzy logic and their main contributions and limitations.
\end{abstract}

\section{KEYWORDS}

Question-answering, Neutral language processing, Fuzzy Logic, Answer Extraction, Evaluation Metrics

\section{INTRODUCTION}

As technology and human-computer interaction advances, there is an increased interest in affective computing and a large amount of data is generated and made it available every day, and for that it requires to integrate and query a huge amount of heterogeneous data, for that NLP has been recognized as a possible solution that capable to manipulate and represent the complex query as uncertain and complicated that are existing in them. Which leads to the generation of QA consisting of Equation and Answer that mapping between these information [1]. However, Question Answering (QA) is a fast-growing research area that combines the research from Information Retrieval, Information Extraction and NLP. It can be seen as the next step in information retrieval is to automatically generating answers to natural language questions from humans, that allow users to pose questions in natural language and receive succinct answers. 
In other aspect (view) Meaning is essential to human language. The idea of the Fuzzy Is use fuzzy set theory that it derived from it a form of multivalued logic to deal with approximate reasoning, to represent and process the linguistic information and attributes of the real world [1]. This survey provides an overview of QA system its system architecture and the possible relationship and differentiation with fuzzy logic, our believe is to have human-friendly dealing with more accuracy in information that needs to use fuzzy logic with NLP.

\section{BACKGROUND}

Over the past four decades Question Answering systems have been transitional much at par with the whole of natural language processing. In this section, we present a previous work on development of QA system and it's propose, the earliest system was developed in 1959 (in the spirit of the era called The Conversation Machine), and A large number of QA system have been developed since 1960's One of the most memorable systems was BASEBALL developed by (Green et al. 1961 in NL DB systems) [2]. Although, capable of answering rather complex questions, BASEBALL was, not surprisingly, for answering questions about baseball games played in the American league over one season, restricted to questions about baseball facts, and most question answering systems were for a long time restricted to front-ends to structured databases. And in 1963 they develop QA system PROTOSYNTHEX that permit user to ask a question in English, it accept statements in (sub set of English) as input to its database and accepts quotations as a query to the database. And for read and solve the kind of word problems they develop (Problem-solving systems) STUDENT QAs system in 1964 That can read and solve the kind of word problems found in high school algebra books. The program is often cited as an early accomplishment of $\mathrm{AI}$ in natural language processing [3].

In early 1966 they provide QA system ELIZA that can communication with user) And This conversation can be simulated with a psychologist. It was able to converse on any topic by resorting to very simple rules that detected important words in the person's input, And in 1972 they develop SHRDLU that offered the possibility to operation of a robot in a toy world (the "blocks world)to be simulated with ability to ask the robot questions about the state of the world SCHOLAR QA system develop in 1973 it was a formal theory characterizing the variety of plausible inferences people use to ask questions about which their knowledge is incomplete. it has methods embed to lessons learned from such research into the SCHOLAR system [4].

In the same year 1973 they develop the first automatic question answering system (SAM). After three years in 1976 TRIPSYS (HWIM) was the first QA that understand speech Question , TRIPSYS(HWIM) was developed as the context for a research project in continuous speech understanding- it understands and answers questions about planned and taken trips, travel budgets and their status, costs of various modes of transportation to various places it's called HWIM (for "Hear What I Mean[2]). And the second famous QA system was (NL DB systems) Problem solving systems LUNAR in 1977[5] for answering questions about the geological analysis of rocks returned by the Apollo moon missions.

In 1977 develop two QA system the first one is GUS it was a dialog system for airline reservation second one was LIFER that develop to asking questions about U.S. Navy ships [6]. This system used a semantic grammar with domain information built within in 1978 they start to develop system that deal with story comprehension (NL DB systems) QUALM an application that use for story comprehension and this application responsible for scripts and plans in a very restrictive 
domain. In 1983 Salton and McGill describe question answering (QA) systems as mainly provide direct answers to questions. Finally Kupiec (1993) employed similar but rather simpler WH question models to build a QA system [8].

In 1991 was QA system (LILOG) text understanding system that operated on the domain of tourism information in a German city. In 1993 they start to combined NLP with the use of an online encyclopedia by developing MURAX QA system that combined NLP with the use of an online encyclopedia with hand-coded annotations to sources. In subsequent developments, QAS aimed on making linguistic analysis of the questions to capture the intended requirements in a natural way [7]. In subsequent developments, one of QAS aimed to capture the intended requirements in a natural way IS to making linguistic analysis of the questions.

In recent 90's, question answering achieved a great progress due to the introduction of the Text Retrieval Conferences (TREC) question answering track there has been great progress in open domain Question answering (Voorhees 2001). These systems use unrestricted text as a primary source of knowledge. One such system, MASQUE (ANDROUTSOPOULOS et al., 1993) use logic representation to represents natural language questions, and for retrieving intended information from database, it use to translates the logic query into a database query. It separates from mapping process the task of linguistic process. FAQ Finder (Burke et al., 1997) does matching of the question list that compiled in a knowledge base with questions through statistical similarity and semantic similarity and for syntax-based natural language understanding technique. In 1999 was LASSO that win the question answering task. It used question classification technique and syntaxbased natural language understanding technique. Another QAS in (2000) was developed by Riloff and Thelen (QUARC), that has the ability to classify questions into different types and use lexical and semantic clue to derive their expected answer [9] [10].

Later, the focus of developing QASs was shifted toward open domain QAS, TREC Evaluation campaign which is taking place every year since 1999 to manage and query large volume of data and represent most of research in open domain question answering from unstructured data sources, and that lead to question-answering evaluations as a recent success started as part of the Text Retrieval Conference (TREC). The best systems are now able to answer more than two thirds of factual questions in this evaluation, it descripts the results and the associated evaluation Methodology develop by Ellen Voorhees, the second paper, by Buchholz and Daelemans, explores the requirements for answering complex questions that have compound answers or multiple correct answers [11].

The third paper, by Lin and Pantel, describes a new algorithm to capture paraphrases that allow a more accurate mapping from questions to potential answers. The fourth paper, by Light, Mann, Rilo and Breck, describes experiments that systematically factor and assess question answering into component sub-problems. The first TREC evaluation campaign provides a list of 200 questions and a document collection. The answers were known to be present in the collections. The maximum lengths of answers were allowed to be 50 or 250 characters[12]. Systems were asked to give 5 ranked lists of answers.

In the next campaign, TREC-9 held in 2000, the number of questions and size of document collections were increased. In TREC-10 in 2001, a new complexity with respect to answers. The lengths of answers were reduced to 50 words [11]. In TREC- 11, held in 2002, systems were expected to give exact short answers to the questions. In TREC from 2002 to 2007, the list of questions, definition questions, and factoid questions were included in the evaluation campaigns. 
In TREC 2005 and TREC 2006 there was temporal question in addition to 75 topics that contains different type of Questions. In TREC 2007, there were a collection of documents that include collection of blogs, and progress competitions that lead to increasing documents collection complexity, and questions complexity, and that also effect to increasing answer evaluation strategies complexity.

In 2000 they start to develop QA system for Unix operating system Unix Consultant For answered questions pertaining to the Unix operating system [10]. This system had ability to accommodate various types of users by phrasing the answer, and it has its domain that contain a comprehensive hand-crafted knowledge base. In 2001 there was two QA systems the first one was INSIGHT question answering system which uses some surface patterns, wins the question answering task in TREC-10, and the second one was SiteQ use the density-based extraction method to retrieve related passages first and then extract the exact answers in them, which can greatly improve the extraction speed.

In 2002 STARTQA system was the first web-based QA system for English and in the same year 2002 was Answer bus QA system(ODQA) they develop this system to accepts questions in several languages (extend the answer extraction process from the local data source to the World Wide Web, which allows them to deal with large count of questions. After one year they develop QA system (ARANEA) it was first fully downloadable open-source Web-based factoid question answering system, and in the same year 2003 create a QA system AQUA was a sophisticated automatic question answering system, which combines natural language understanding technique, ontological knowledge, logical reasoning abilities and advanced knowledge extraction techniques [12].

In 2008 they start to develop a new QA system with different approach and it was a List questions ask for different instances of a particular kind of information to be returned.in 2012 they develop QA Systems that oriented to work with opinions rather than facts can also be included in this category. In 2014 was QAKIS by Cabrio et al and FREITAS14 develop by Freitas and Curry and also INTUI3 by Dima, in 2015 was develop three QA system first was HAKIMOV15 by Usbeck et al [12]. and second was QASYO by Hakimov et al. last. However, QA systems have developed over the past few decades until they reached the structure that we have nowadays.

\subsection{Implementation Approaches for QA Systems}

The basic aim of QA System is to provide correct and short answer with high accuracy to the user and There are many Approaches used in Question answering system based on different purpose [13]. This section will present an implementation approaches for various categories of QA System such as:

\subsubsection{First Approach}

That relied on artificial intelligence (AI) it called Linguistic approach and it has the ability to build QA logics by using methods that integrated natural language processing (NLP) techniques and knowledge base. It used Linguistic techniques for formulating user's question into a precise query that merely extracts the respective response from the structured database, it Used to understand natural language text, linguistic \& common knowledge Linguistic techniques such as such as (tokenization, POS tagging and parsing [14]. 


\subsubsection{Second Approach}

(rule-based approach) that rely on the (rule-based mechanism), they are built to identify question classification features. Quarc developed by Rilloff et al., and Cqarc [15] developed by Hao et al. Used to identify the question class by using semantic clues in question and for looking lexical they used heuristic rules.

\subsubsection{The Third Approach}

(Statistical approach) gives the better results than other approaches user can use this approaches successfully to applied the different stages of a QA system, it independent of structured query languages and can formulate queries in natural language form. IBM's statistical QA system was based on the statistical model. This system utilized maximum entropy model for question/ answer classification based on various N-gram or bag of words features. Moschitti had used Rocchio and SVM text classifiers for question and answer categorization. Availability of huge amount of data on internet increased the importance of statistical approaches [16].

\subsubsection{The Fourth Approach}

is Pattern matching that have the ability to replace the sophisticated processing involved in other competing approaches by using the expressive power of text patterns. Some of patterns matching QA rely on templates for response generation while most of of the patterns matching QA systems [17].

\subsubsection{The Fifth Approach}

is the Surface Pattern based approach. This approach rely on an extensive list of patterns to extracts answers from the surface structure of the retrieved documents. It's automatically learning based pattern or it is human crafted. [18]. However, to identify any question answer it depend on the basis of similarity between their reflecting patterns having certain semantics as well as their Methods, definition and Characteristics, Limitation and Aims for each QA system that was developed before which will be helpful for new directions of research in this area (Table 1).

Table 1: List of Most Popular QA Systems

\begin{tabular}{|c|c|c|c|}
\hline 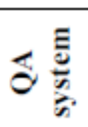 & Method \& definition & Characteristics and Implementation Issues & Limitation \\
\hline $\begin{array}{l}\bar{a} \\
\frac{a}{\infty} \\
\frac{\pi}{\infty} \\
\frac{\pi}{\infty}\end{array}$ & $\begin{array}{l}\text { Description: answers English } \\
\text { questions-about-the scores, } \\
\text { teams, locations, and dates of } \\
\text { baseball games. } \\
\text { Method: Analyzed the } \\
\text { question, using-linguistic } \\
\text { knowledge-, into canonical } \\
\text { form. }\end{array}$ & $\begin{array}{l}\text { Characteristics: Input sentences have to be simple, and } \\
\text { not contain-sentential- connectives, such as (and, or). } \\
\text { - The data are stored in a data base in attribute-value } \\
\text { format. - questions-transformed into the same format, } \\
\text { but in an automatic way. } \\
\text { Implementation: system was implemented in the } \\
\text { Lincoln Laboratory. And it relied on Linguistic } \\
\text { approach. }\end{array}$ & $\begin{array}{l}\text { - its domain of \{baseball } \\
\text { only\} (Close domain) } \\
\text {-the database tied to } \\
\text { specific domains, where } \\
\text { the attribute-value } \\
\text { structures can be } \\
\text { uniform, and the types } \\
\text { of questions are limited. }\end{array}$ \\
\hline $\begin{array}{l}\frac{\overline{2}}{\bar{n}} \\
\frac{1}{0} \\
\frac{1}{n}\end{array}$ & $\begin{array}{l}\text { Description: Correctly } \\
\text { solving the algebra problem } \\
\text { Method: was taken-as-a } \\
\text { demonstration-that the system } \\
\text { understood the written } \\
\text { statement of the problem. }\end{array}$ & $\begin{array}{l}\text { characteristics: Understanding-language-requires- } \\
\text { world-knowledge. } \\
\text {-read and solved high school algebra word problems. } \\
\text { Implementation : It is written in Lisp }\end{array}$ & $\begin{array}{l}\text { - systems were limited } \\
\text { by the amount of } \\
\text { knowledge-they } \\
\text { contained. } \\
\text { Closed Domain }\end{array}$ \\
\hline
\end{tabular}




\begin{tabular}{|c|c|c|c|}
\hline 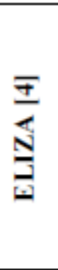 & $\begin{array}{l}\text { Description: system that } \\
\text { simulated a Conversation with } \\
\text { a psychologist. } \\
\text { Method: the computer can } \\
\text { read messages typed on the } \\
\text { typewriter and respond by } \\
\text { writing on the same } \\
\text { instrument. }\end{array}$ & $\begin{array}{l}\text { Characteristics: ability to converse on any topic by } \\
\text { resorting to very simple rules that detected important } \\
\text { words in the person's input. } \\
\text { Implementation: it was first implemented in the SLIP } \\
\text { language, that use as an extension to FORTRAN but with } \\
\text { better functionality to process doubly linked lists, and Its } \\
\text { present implementation is on the MAC time-sharing } \\
\text { system at MIT. And relied on Linguistic approach. }\end{array}$ & $\begin{array}{l}\text { - (closed domain) the } \\
\text { knowledge stored in the } \\
\text { structured database was } \\
\text { only capable of } \\
\text { answering questions } \\
\text { asked within the } \\
\text { restricted domain. }\end{array}$ \\
\hline 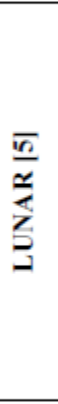 & $\begin{array}{l}\text { Description: Designed to } \\
\text { enable a lunar geologist to } \\
\text { conveniently access the } \\
\text { chemical analysis data on lunar } \\
\text { rock that was accumulating as } \\
\text { a result of the Apollo moon } \\
\text { mission". } \\
\text { Method: The entries in the } \\
\text { analysis table-specify-the } \\
\text { concentration of some } \\
\text { constituent in some phase of } \\
\text { some sample. }\end{array}$ & $\begin{array}{l}\text { characteristics: -The system contains two data bases: a } \\
13,000 \text {-entry table off chemical and age analyses of the } \\
\text { Apollo } 11 \text { samples. } \\
\text { - able to answer } 90 \% \text { of the in-domain questions posed } \\
\text { by working geologists, without prior instructions as to } \\
\text { phrasing. } \\
\text { implementation system is implemented in LISP. Parse } \\
\text { English question into a data base query, Syntactic } \\
\text { analysis via augmented transition network parser and } \\
\text { heuristics and relied on Linguistic approach. That can } \\
\text { analyze automatically with a transition network parser } \\
\text { and translated into a data base query language. }\end{array}$ & $\begin{array}{l}\text {-Sophisticated, with the } \\
\text { syntax and semantics of } \\
\text { questions } \\
\text { - having a particular } \\
\text { database could not be } \\
\text { easily modified to be } \\
\text { used with different } \\
\text { databases. } \\
\text {-closed domain }\end{array}$ \\
\hline$\frac{5}{5}$ & $\begin{array}{l}\text { Description : Simulated a } \\
\text { travel advisor. } \\
\text { Method: it attempt to explore } \\
\text { the integration of already } \\
\text { existing programming } \\
\text { technology for a performance } \\
\text { demonstration. }\end{array}$ & $\begin{array}{l}\text { characteristics: had access to a restricted database of } \\
\text { information about airline flights. } \\
\text { - is a steady genetic algorithm with subpopulation } \\
\text { support. } \\
\text { Implementation: system implemented in MChART, it } \\
\text { is a framework for implementing parsers. And relied on } \\
\text { Linguistic approach. }\end{array}$ & $\begin{array}{l}\text {-closed domain } \\
\text {-the knowledge stored } \\
\text { in the structured } \\
\text { database was only } \\
\text { capable of answering } \\
\text { questions asked within } \\
\text { the restricted domain. }\end{array}$ \\
\hline 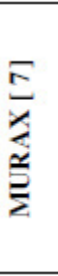 & $\begin{array}{l}\text { Description: searched the } \\
\text { encyclopedia for noun Phrases } \\
\text { identified from the question. } \\
\text { Method: exploited the phrase } \\
\text { relations contained in the } \\
\text { question to match questions } \\
\text { with answer hypotheses. }\end{array}$ & $\begin{array}{l}\text { Characteristics: } \\
\text {-used the technology of robust shallow parsing. } \\
\text {-use the Internet as a corpus } \\
\text { - answers hypothesize noun phrases } \\
\text { Implementation: the sentences of matching text are } \\
\text { selected to confirm phrase relations implied by the } \\
\text { question, rather than being selected solely on the basis } \\
\text { of word frequency. }\end{array}$ & $\begin{array}{l}\text {-lack of basic- } \\
\text { information extraction } \\
\text { support } \\
\text {-open domain }\end{array}$ \\
\hline $\begin{array}{l}\frac{\infty}{2} \\
\frac{1}{2} \\
\vdots\end{array}$ & $\begin{array}{l}\text { Description: designed to test a } \\
\text { story understanding system. } \\
\text { Method: -it requires question } \\
\text { to be parsed and a conceptual } \\
\text { graph to be built, and it include } \\
\text { two stages: understanding the } \\
\text { question and finding the } \\
\text { answer. }\end{array}$ & $\begin{array}{l}\text { Characteristics: it able to answer questions about } \\
\text { ideas not specifically mentioned in the texts. } \\
\text { - represented as a conceptual graph and text } \\
\text { comprehension } \\
\text { Implementation: - it implemented as a language- } \\
\text { independent question answering module, and } \\
\text { integrated into other natural language processing } \\
\text { applications }\end{array}$ & $\begin{array}{l}\text {-hard to classify and } \\
\text { complex system } \\
-\quad \text { (closed domain) } \\
\text { highly restricted in } \\
\text { terms of the domain, or } \\
\text { world, knowledge } \\
\text { required and the genre } \\
\text { of text covered }\end{array}$ \\
\hline 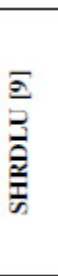 & $\begin{array}{l}\text { Description: it's a research } \\
\text { system that help researchers } \\
\text { understand the issues involved } \\
\text { in modeling human dialogue. } \\
\text { Method: the system answers } \\
\text { questions, execute commands, } \\
\text { and accepts information in } \\
\text { normal English Dialog. }\end{array}$ & $\begin{array}{l}\text { characteristics: contain a combination of syntax, } \\
\text { semantics, and reasoning. } \\
\text { - it uses semantic information and context to } \\
\text { understand discourse and to disambiguate sentences. } \\
\text {-it could search back further through the interactions. } \\
\text { implementation : its written in Micro Planner and } \\
\text { Lisp ( a general-purpose, multi-paradigm } \\
\text { programming language. }\end{array}$ & $\begin{array}{l}\text {-constrained to a simple } \\
\text { block world. } \\
\text {-restricted-domain } \\
\text { - has a core database } \\
\text { handwritten by experts }\end{array}$ \\
\hline $\begin{array}{l}\frac{\Xi}{\varrho} \\
\frac{n}{2} \\
\frac{\sigma}{2}\end{array}$ & $\begin{array}{l}\text { Description allows end users to } \\
\text { submit a query to an RDF triple } \\
\text { store in English and obtain the } \\
\text { answer in the same language. } \\
\text { Method: it composed of two } \\
\text { main modules the query } \\
\text { generator takes the user } \\
\text { question as input, generates the } \\
\text { typed questions, and then } \\
\text { generates the SPARQL } \\
\text { queries from the retrieved } \\
\text { patterns. }\end{array}$ & $\begin{array}{l}\text { Characteristics: huge amounts of available semantic } \\
\text { data } \\
\text {-implement a relation-based match for question } \\
\text { interpretation, to convert the user question into a } \\
\text { query language } \\
\text { Implementation: QAKiS addresses the task of QA } \\
\text { over structured Knowledge Bases (KBs) where the } \\
\text { relevant information is expressed in unstructured form } \\
\text { to implement a relation-based match for question } \\
\text { interpretation, to convert the user question into a } \\
\text { query language. }\end{array}$ & Open domain \\
\hline
\end{tabular}




\begin{tabular}{|c|c|c|c|}
\hline 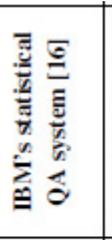 & $\begin{array}{l}\text { Description:it's IBMstatically } \\
\text { question answer for TRCE 9.Is } \\
\text { an application of maxim } \\
\text { entropy classification for QA } \\
\text { predication and name entity } \\
\text { marking } \\
\text { Method: maxim entropy }\end{array}$ & $\begin{array}{l}\text { characteristics: classification based on various N- } \\
\text { gram or bag of words features. } \\
\text { - Trained on crops of DSTS--use REASON } \\
\text { Implementation: Maximum Entropy Model for this } \\
\text { (IBM's QA) system used a two-pass approach based } \\
\text { on Okapi formula and expansion of queries based on } \\
\text { TREC-9 QA corpus. -Statistical approach based }\end{array}$ & Open domain \\
\hline 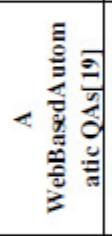 & $\begin{array}{l}\text { Description: presents an } \\
\text { answer extraction method } \\
\text { which based on the calculation } \\
\text { of sentence similarity between } \\
\text { question and answer. (Sentence } \\
\text { Similarity Model) }\end{array}$ & $\begin{array}{l}\text { characteristics: using Web data resource as database } \\
\text { for question answering system } \\
\text { - use answer correctness as our evaluation me } \\
\text { Implementation: Statistical models applied for } \\
\text { question classification }\end{array}$ & $\begin{array}{l}\text {-Open domain } \\
\text {-difficulty of finding } \\
\text { answers to questions } \\
\text { little data available in } \\
\text { the target language in } \\
\text { which to search for } \\
\text { answers. }\end{array}$ \\
\hline 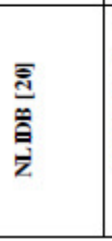 & $\begin{array}{l}\text { Description: it's a database } \\
\text { system Engaged the user in } \\
\text { dialogues, it allows the user to } \\
\text { access information stored in a } \\
\text { database. }\end{array}$ & $\begin{array}{l}\text { characteristics: used with large databases. } \\
\text {-could be configured to interface to different } \\
\text { underlying database. Implementation: it was } \\
\text { implemented entirely in Prolog. It transformed } \\
\text { English questions into Prolog expressions, which were } \\
\text { evaluated against the Prolog database. The code of } \\
\text { Chat- } 80 \text { was circulated widely) }\end{array}$ & $\begin{array}{l}\text { Closed Domain } \\
\text { the knowledge stored in } \\
\text { the structured database } \\
\text { was only capable of } \\
\text { answering questions in } \\
\text { restricted domain. }\end{array}$ \\
\hline 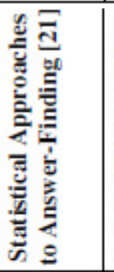 & $\begin{array}{l}\text { Description: investigates } \\
\text { whether a machine can } \\
\text { automatically learn the task of } \\
\text { finding, within a large } \\
\text { collection of candidate } \\
\text { responses } \\
\text { Method: use four statistical } \\
\text { techniques for answer-finding }\end{array}$ & $\begin{array}{l}\text { characteristics- Usenet FAQ documents and } \\
\text { customer service call-center dialogues from a large } \\
\text { retail company } \\
\text { Implementation - statistical models applied for } \\
\text { question classification. } \\
\text { using, adaptive tf. idf algorithm and automatic query } \\
\text { expansion with latent variable model. }\end{array}$ & Open domain \\
\hline 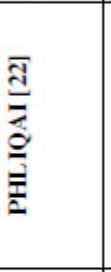 & $\begin{array}{l}\text { Description: it Designed to } \\
\text { answer short questions against } \\
\text { a data base containing fictitious } \\
\text { data about computer } \\
\text { installations in Europe and } \\
\text { companies using them. } \\
\text { Method: - use of the lambda } \\
\text { calculus }\end{array}$ & $\begin{array}{l}\text { Characteristics: Have three division of the } \\
\text { translation from natural language questions to data } \\
\text { base 1-English-oriented Formal Language (EFL). 2- } \\
\text { World Model Language (WML). } \\
\text { 3-Data Base Language (DBL). } \\
\text { Implementation: in PHLIQAl system Questions are } \\
\text { translated into a formal language used to access the } \\
\text { data base. }\end{array}$ & $\begin{array}{l}\text { the limitation to a } \\
\text { narrow domain }\end{array}$ \\
\hline 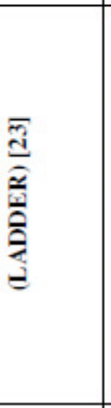 & $\begin{array}{l}\text { Description: it's a database } \\
\text { systems It was designed as a } \\
\text { natural language interface to a } \\
\text { database of information about } \\
\text { US Navy ships. } \\
\text { Method: used semantic } \\
\text { grammars to parse questions to } \\
\text { query distributed database. it } \\
\text { candeveloped as a prototype } \\
\text { system for understanding } \\
\text { questions posed in English } \\
\text { about a naval domain. }\end{array}$ & $\begin{array}{l}\text { Characteristics: used with large databases . } \\
\text { - grammar must be tailor-made for each given } \\
\text { database. } \\
\text { - only support simple one table queries or multiple } \\
\text { table queries. } \\
\text { Implementation: used a semantic grammar to parse } \\
\text { questions and query a distributed database.it translated } \\
\text { each English question into one or more relational } \\
\text { database queries, prosecuted the queries on a remote } \\
\text { computer.Based on LIFER parser, which interpreted } \\
\text { sentences according to a 'semantic grammar." }\end{array}$ & $\begin{array}{l}\text {-a different grammar } \\
\text { had to be developed } \\
\text { whenever Ladder was } \\
\text { configured for a new } \\
\text { application. } \\
\text { - a narrow domain }\end{array}$ \\
\hline 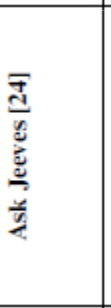 & $\begin{array}{l}\text { Description : allowed end- } \\
\text { users to teach the system new } \\
\text { words and concepts at any point } \\
\text { during the interaction it's } \\
\text { database systems } \\
\text { method: The user stated } \\
\text { requests in English, and Ask } \\
\text { suitable requests to the } \\
\text { appropriate underlying systems. }\end{array}$ & $\begin{array}{l}\text { Characteristics: has its own built-in database. } \\
\text { - use the Internet as a corpus } \\
\text { Implementation : its pointing the questioner to Web } \\
\text { links that might contain information relevant to the } \\
\text { answer to the question. }\end{array}$ & $\begin{array}{l}\text { All the applications } \\
\text { connected to Ask were } \\
\text { accessible to the end- } \\
\text { user through natural } \\
\text { language requests } \\
\text {-open domain }\end{array}$ \\
\hline
\end{tabular}




\begin{tabular}{|c|c|c|c|}
\hline 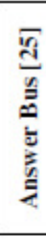 & $\begin{array}{l}\text { Description:is an question } \\
\text { answering system based on } \\
\text { sentence level Web information } \\
\text { retrievalmethod : Answer Bus } \\
\text { extracts sentences that are } \\
\text { determined to contain answers. }\end{array}$ & $\begin{array}{l}\text { Characteristics: High performance \& accuracy for } \\
\text { response time.-use the Internet as a corpus. } \\
\text {-It accepts questions in several languages. } \\
\text { Implementation: is based on sentence level } \\
\text { information retrieval. It accepts users' natural- } \\
\text { language questions in different language and extracts } \\
\text { possible answers from the Web. }\end{array}$ & $\begin{array}{l}\text {-it only extracts the } \\
\text { named entities that } \\
\text { match question types } \\
\text {-open-domain }\end{array}$ \\
\hline$\frac{\sqrt[3]{3}}{3}$ & $\begin{array}{l}\text { Description: allow users to ask } \\
\text { questions in everyday language } \\
\text { and receive an answer quickly } \\
\text { and with a context } \\
\text { Method: Use a similarity } \\
\text { algorithm to map between } \\
\text { names of relations in the } \\
\text { knowledge base and names of } \\
\text { relations in the ontology }\end{array}$ & $\begin{array}{l}\text { Characteristics: it use semantic annotations to } \\
\text { perform inferences. } \\
\text { - AQUA's inference engine operates within the } \\
\text { framework of multi-sorted logic, } \\
\text { Implementation: it uses NLP technology, Logic and } \\
\text { a hand-crafted ontology. And using Dice coefficient } \\
\text { and WordNet algorithm. This algorithm is used to } \\
\text { ensure that the question does not fail. }\end{array}$ & Closed domain \\
\hline$\frac{\sqrt{a}}{\sqrt{a}}$ & $\begin{array}{l}\text { Description: it's a First Web- } \\
\text { Based Question Answering } \\
\text { System, Designed To Answer } \\
\text { Questions That Are Posed To- } \\
\text { It-In-Natural Language. } \\
\text { method: It Parses Incoming } \\
\text { Questions, Matches the Queries } \\
\text { Created from The Parse Trees } \\
\text { Against Its Knowledge Base } \\
\text { And Presents-The Appropriate- } \\
\text { Information Segments To The } \\
\text { User. }\end{array}$ & $\begin{array}{l}\text { Characteristics Ability to Answer millions of } \\
\text { English-Questions-- use the Internet as a corpus } \\
\text { - used technique called "natural language annotation" } \\
\text { Implementation consists of two modules. } \\
\text { The understanding module: analyzes English text and } \\
\text { produces a knowledge base that encodes information } \\
\text { found in the text. } \\
\text {-the generating module: produces English sentences. } \\
\text { Used in conjunction with the technique of natural } \\
\text { language annotation. } \\
\text {-Linguistic based QA systems }\end{array}$ & $\begin{array}{l}\text {-ambiguous } \\
\text { modification } \\
\text { linguistically- } \\
\text { uninformed QA } \\
-\quad \text { have difficulty } \\
\text { handling-semantic } \\
\text { symmetry } \\
\text { - open domain }\end{array}$ \\
\hline
\end{tabular}

Table2: Fuzzy Logic Implementation Approaches For QA Systems

\begin{tabular}{|c|l|}
\hline \multicolumn{1}{|c|}{ QA System } & \multicolumn{1}{c|}{ Method \& Definition } \\
\hline $\begin{array}{c}\text { Quantitative Fuzzy Semantics } \\
\text { (L. A. ZADEH)(1971) }\end{array}$ & $\begin{array}{l}\text { the first paper on fuzzy sets it can construct } \\
\text { fuzzy query languages for purposes of } \\
\text { information retrieval, and, possibly, to } \\
\text { implementation of fuzzy algorithms and } \\
\text { programs. [37] }\end{array}$ \\
\hline $\begin{array}{c}\text { A Fuzzy-Set-Theoretic Interpretation of Linguistic } \\
\text { Hedges L. A. Zadeh (1972) }\end{array}$ & $\begin{array}{l}\text { represent a hedge as an operator, linguistic } \\
\text { hedge such as very, more or less, much, } \\
\text { essentially, slightly. [35] }\end{array}$ \\
\hline $\begin{array}{c}\text { PRUF- A Meaning Representation Language For } \\
\text { Natural Languages" (1978) }\end{array}$ & $\begin{array}{l}\text { PRUF provide a basis for question-answering } \\
\text { and inference from fuzzy premises [40]. }\end{array}$ \\
\hline $\begin{array}{c}\text { Test-Score- Semantics as A Basis For A } \\
\text { Computational Approach (1986) }\end{array}$ & $\begin{array}{l}\text { test-score semantics provides a framework for } \\
\text { the representation of the meaning of } \\
\text { dispositions.[42] }\end{array}$ \\
\hline A Computational Approach to Fuzzy Quantifiers In \\
Natural Languages (1983) & $\begin{array}{l}\text { deal with fuzzy quantifiers (denote the } \\
\text { collection of quantifiers in NLP whose } \\
\text { representative elements are: much, not many, } \\
\text { very many, notvery many) that are treated as } \\
\text { fuzzy numbers[37] }\end{array}$ \\
\hline
\end{tabular}




\begin{tabular}{|c|c|}
\hline $\begin{array}{l}\text { Fuzzy Quantifiers: A Natural Language Technique } \\
\text { for Data Fusion } 2001\end{array}$ & $\begin{array}{l}\text { intended to formalize the notion of 'linguistic } \\
\text { adequacy'. then argue that the models of the } \\
\text { theory are plausible from a linguistic } \\
\text { perspective.[37] }\end{array}$ \\
\hline $\begin{array}{l}\text { Fuzzy Logic-Based Natural Language Processing } \\
\text { and Its Application to Speech Recognition } \\
\qquad 2017\end{array}$ & $\begin{array}{l}\text { it create a system that can learn from a } \\
\text { linguistic corpus. it use fuzzy semantic } \\
\text { relations to represented by words and use such } \\
\text { relations to process the word sequences } \\
\text { generated by speech recognition systems. [32] }\end{array}$ \\
\hline $\begin{array}{l}\text { The Fuzzy Formal Concept Analysis (FFCA) } \\
\qquad 2003\end{array}$ & $\begin{array}{l}\text { proposed a fuzzy FCA-based approach for } \\
\text { conceptual clustering for automatic generation } \\
\text { of concept hierarchy from uncertainty } \\
\text { information.[43] }\end{array}$ \\
\hline $\begin{array}{c}\text { A Hybrid Approach Using Ontology Similarity and } \\
\text { Fuzzy Logic } \\
\text { For Semantic Question Answering } 2014\end{array}$ & $\begin{array}{l}\text { the user enters a source string as a question. the } \\
\text { first objective of the machine is to syntactically } \\
\text { analyze the text from the source. using fuzzifier } \\
\text { approach (semantic fuzzy ontology) [44] }\end{array}$ \\
\hline $\begin{array}{c}\text { Improving translation memory fuzzy matching by } \\
\text { paraphrasing } \\
2015\end{array}$ & $\begin{array}{l}\text { present an innovative approach to match } \\
\text { sentences having different words but the same } \\
\text { meaning. presented a method that improves the } \\
\text { fuzzy match of similar, but not identical } \\
\text { sentences. [41] }\end{array}$ \\
\hline $\begin{array}{l}\text { Semantic parsing on freebase from question-answer } \\
\text { pairs }\end{array}$ & $\begin{array}{l}\text { train a semantic parser that scales up to } \\
\text { Freebase.method : build a coarse mapping from } \\
\text { phrases to predicates using a knowledge base } \\
\text { and a large text corpus. [33] }\end{array}$ \\
\hline $\begin{array}{c}\text { FLINTSTONES: A fuzzy linguistic decision tools } \\
\text { enhancement suite based on the 2-tuple linguistic } \\
\text { model and extensions }\end{array}$ & $\begin{array}{l}\text { Use fuzzy linguistic tools called Flintstones } \\
\text { that proposed to solve linguistic decision } \\
\text { making problems based on the 2-tuple } \\
\text { linguistic model in order to validate the } \\
\text { performance of the software suite with real } \\
\text { datasets[34] }\end{array}$ \\
\hline $\begin{array}{l}\text { A fuzzy linguistic approach for human resource } \\
\text { evaluation and selection in software projects } \\
2015\end{array}$ & $\begin{array}{l}\text { present a fuzzy linguistic approach that utilizes } \\
\text { 2-tuple fuzzy linguistic terms and supports the } \\
\text { selection of suitable human resources based on } \\
\text { their skills and the required skills for each } \\
\text { project task.[35] }\end{array}$ \\
\hline $\begin{array}{l}\text { Fuzzy logic in natural language processing } \\
\qquad 2017\end{array}$ & $\begin{array}{l}\text { they outline how model of the meaning of basic } \\
\text { constituents of natural language (nouns, } \\
\text { adjectives, adverbs, verbs) has been elaborated } \\
\text { in FNL[39] }\end{array}$ \\
\hline
\end{tabular}




\section{QA SYSTEM OVERVIEW}

Question answering is a process that understanding user natural language query and has the ability to provide a correct answer and extract it from retrieving relevant documents, data, or knowledge base .A question answering (QA) Question-answering systems are referred to as intelligent systems ,that can be used to provide responses for the questions being asked by the user based on certain facts or rules stored in the knowledge base it can generate answers of questions asked in natural [28].

\subsection{QA System Purpose and Types}

The purpose of a QA system is to provide correct answers to user questions in both structured and non-structured collection of data. there are three main types for the QA Depending on the target domain and the way questions are answered it will present in table.

Table 4: Types of QA Systems

\begin{tabular}{|c|c|c|}
\hline & $\begin{array}{c}\text { TYPES OF QA SYSTEMS } \\
\end{array}$ & ADVANTAGE \\
\hline $\begin{array}{l}z \\
z \\
z \\
0 \\
\vdots \\
0 \\
0 \\
0 \\
0 \\
0 \\
0\end{array}$ & $\begin{array}{l}\text { Built for very specific domains and exploit expert } \\
\text { knowledge. } \\
\text { The first QA systems of this type were developed in the } \\
\text { 1960s. Any data not present in the database is usually } \\
\text { considered out-of-domain. } \\
\text { Closed-domain question answering deals with } \\
\text { questions under a specific domain, This type of QA is } \\
\text { easier for the vocabulary is more predictable, and } \\
\text { ontologies describing the domain are easier to construct } \\
\text {-Two of the most cited closed-domain QA systems are } \\
\text { BASEBALL }{ }^{[2]} \text { and LUNAR }\end{array}$ & $\begin{array}{l}\text { Deals with very specific data which usually } \\
\text { does not contain ambiguous terms and as a } \\
\text { result can be processed more easily. }\end{array}$ \\
\hline $\begin{array}{l}z \\
z \\
\vdots \\
\vdots \\
z \\
\vdots \\
\vdots \\
\vdots\end{array}$ & $\begin{array}{l}\text { Can be asked about virtually any topic and can } \\
\text { theoretically extract the answer from any textual } \\
\text { collection. } \\
\text { - It can deals with unrestricted topics. Hence, questions } \\
\text { may concern any subject. The corpus may consist of } \\
\text { unstructured or structured texts. } \\
\text { - Two of good example open domain QA systems are } \\
\text { AnswerBus }{ }^{[25]} \text { and Askjeeve }{ }^{[24]}\end{array}$ & $\begin{array}{l}\text { An alternative to search engines, available } \\
\text { on the Web. Instead of providing a list of } \\
\text { relevant keywords user just asks a } \\
\text { question. } \\
\text {-Automatically build queries and retrieve } \\
\text { relevant documents, extract answers from } \\
\text { the retrieved text snippets and present } \\
\text { them as a confidence-ranked list to the } \\
\text { user. }\end{array}$ \\
\hline
\end{tabular}

\section{QA SYSTEM ARCHITECTURE \& ALgORITHM}

\subsection{System Architecture}

As shown in figure (1) a QA system contain three main part beside other supplementary components: question classification (QUESTION PROCESSING), information retrieval (DOCUMENT PROCESSING) and answer extraction (ANSWER PROCESSING). The user writes a question using the user query interface. Then this query is used to extract all the possible 
answers for the input question. The architecture of Question Answering system is as shown in Figure1

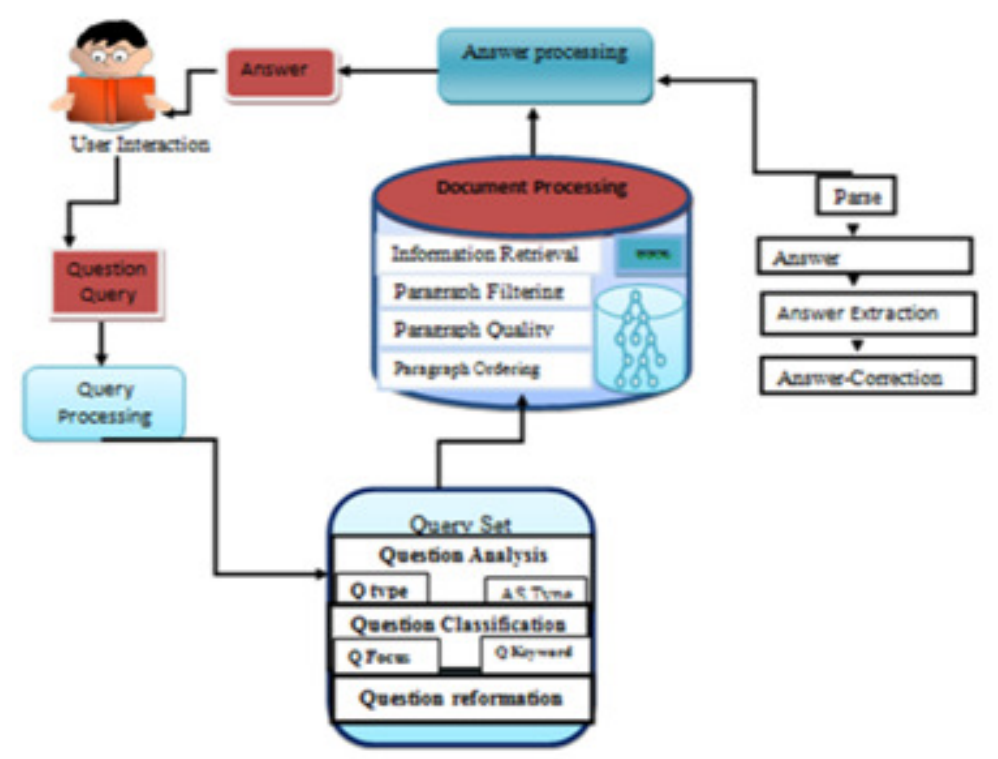

Figure (1): Question Answering System Architecture

\subsection{Question Answering System Algorithm}

\subsubsection{Step One}

(Question processing): Given a question as input, user writes his question by an interface the function of the question processing module is to process and analyses the question, and to create some representation of the information requested module is to process and analyses the question, and to create some representation of the information requested the question, and to create some representation of the information requested analyses the question, and to create some representation of the information requested. This leads to the classification of question that include three parts: Query Interface, question analyzer and Question classification. This step help to generate complex structured queries . and detects the expected answer type of a question e.g. the expected answer type of " When was last time that you feel this headache?" is date or "Do other family members have similar headaches?" Is yes/no this information helps guide the answer extraction process [29].

\subsubsection{Step Two}

after Input questions -Determining the question type: is selected from question taxonomy that system uses. and candidate answers are extracted using all the information gathered in the previous steps, e.g., keywords, entities and relations. The remaining candidate answers are then filtered and ranked according to different measures of similarity to filter out candidates with incompatible types. The Document Processing Module retrieves documents from the corpus that are likely to contain answers to the user's question. It consists of a query generation algorithm, text search engine and information retrieval that retrieve the relevant documents based upon important keywords appearing in the question. The query generation algorithm takes an input the 
user's question and creates a query containing terms likely to appear in documents containing an answer. This query is passed to the text in system, which uses it to retrieve answer. [30].

\subsubsection{Step Three}

The answer processing module is responsible for identifying, extracting and validating answers from the set of ordered paragraphs passed to it from the Document Processing Module, takes input from the retrieval component and tries to retrieve an exact phrase to return as an answer to achieve this required parsing and detailed question analysis by using of answer extraction algorithms. The identity answer extraction returns the CenterPoint of the passage, stripping words from either end until it fits within the specified answer. Then Answer Display The result and converted into required text which is required by the user and displayed to the user.

\subsection{Evaluation Metrics}

There are several parameters that are used to analyze the performance of different Question Answering Systems. In this section we describe some of the evaluation metrics used in Question Answering System to evaluate its performance such as: precision, recall and F-measure, a weighted harmonic mean of precision and recall, can be defined with respect to predicates for the purposes of QA evaluation. These precisions and recall metrics express true precision and recall, not approximations, when coupled with an answer key in which the judgments can be reasonably assumed to be exhaustive[31].

\subsubsection{Precision}

Precision- Recall is the most common metric to evaluate information retrieval system. Precision is the ratio of retrieved documents that are relevant to all retrieved documents in the ranked list $[30]$.

$$
\text { precision }=\frac{\mid\{\text { relevant documents }\} \cap\{\text { retrieved documents }\} \mid}{\mid\{\text { retrieved documents }\} \mid}
$$

\subsubsection{Recall}

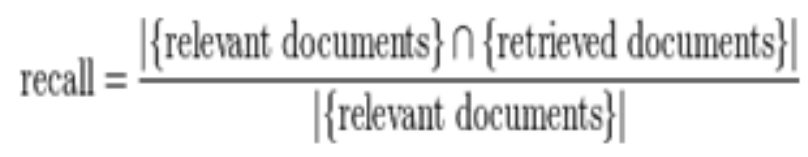

Recall in information retrieval is the fraction of the documents that are relevant to the query that are successfully retrieved.

For example: text search on a set of documents recall is the number of correct results divided by the number of results that should have been returned In binary classification, recall is called sensitivity. So it can be looked at as the probability that a relevant document is retrieved by the query. It is trivial to achieve recall of $100 \%$ by returning all documents in response to any query[36]. Therefore, recall alone is not enough but one needs to measure the number of nonrelevant documents. 


\subsubsection{F-Measure}

A measure that combines precision and recall is the harmonic mean of precision and recall, the traditional F-measure or balanced F-score:

$$
F=2 \cdot \frac{\text { precision } \cdot \text { recall }}{\text { precision }+ \text { recall }}
$$

known as the $F_{1}$ measure, because recall and precision are evenly weighted. It is a special case of the general $F_{\beta}$ measure (for non-negative real values of $\beta$ ):

$$
F_{\beta}=\left(1+\beta^{2}\right) \cdot \frac{\text { precision } \cdot \text { recall }}{\beta^{2} \cdot \text { precision }+ \text { recall }}
$$

Two other commonly used $F$ measures are the $F_{2}$ measure, which weights recall higher than precision, and the $F_{0.5}$ measure, which puts more emphasis on precision than recall.

The F-measure was derived by van Rijsbergen (1979) so that $F_{\beta^{\prime \prime}}$ measures the effectiveness of retrieval with respect to a user who attaches $\beta$ times as much importance to recall as precision"[36].

It is based on van Rijsbergen's effectiveness measure $E=1-\frac{1}{\frac{a}{P}+\frac{1-\alpha}{R}}$

Their relationship is $F_{\beta}=1-E_{\text {where }} \alpha=\frac{1}{1+\beta^{2}}$

\section{FUZZY LOGIC OVERVIEW}

This section presents a quick overview of what fuzzy logic is and its system architecture and the possible relationship with Question Answer, Fuzzy logic is a technique used for representing and manipulating uncertain information, In the more traditional propositional logic, each fact or proposition, the truth vale in fuzzy logic may range between completely true and completely false that it's a concept of partial truth that fuzzy logic used to handle it By contrast, in logic. non-numeric values are often used to facilitate the expression of rules and facts linguistic variable and that in fuzzy logic application such as age may accept values such as young and its antonym old. And Because natural languages do not always contain enough value terms to express a fuzzy value scale, it is common practice to modify linguistic values with adjectives or adverbs[37].

So any systems that cannot be precisely described by mathematical models or devices that have significant uncertainties or contradictory conditions, and linguistically controlled devices or systems can use fuzzy logic. As Lotfi Zadeh once stated, fuzzy logic is not going to replace conventional logic or methodologies, rather it will supplement them in circumstances where conventional approaches fail to solve a problem effectively [38]. In recent years, Fuzzy logic has proved to be particularly useful in expert system and other artificial intelligence applications and NLP . Therefore, many of the previous researchers in academia, applications include modeling, evaluation, optimization, decision making, control, diagnosis and information Measure the 
growing interest in fuzzy logic. In particular, fuzzy logic is best suited for control-systems fields. it has been applied in areas such as breakdown prediction of nuclear reactors in Europe, earthquake forecasting in China, and subway control in Japan.

\subsection{Fuzzy Logic General Architecture}

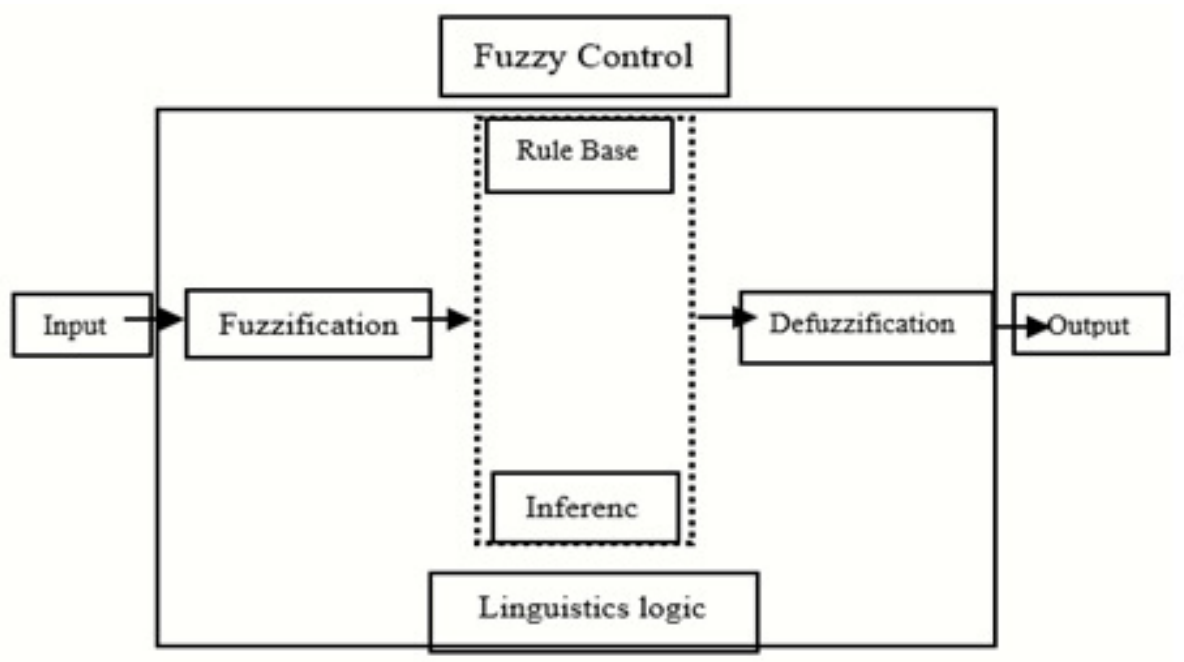

Figure 2: Fuzzy Logic General Architecture

\subsection{1-Fuzzification}

receives the inputs and transforms it into fuzzy sets (by maps the given inputs to fuzzy sets and linguistic variable). the fuzzy rules premises that are predefined in the rule base of the specified application can be matched with defined linguistic variables [36].

\subsection{2- Rule Base}

includes a set of linguistic rules designed in the form (if-then rules). These rules define the consequent of the model in terms of the given linguistic variables such as low, moderate and high. In addition, it specifies the type and number of the used membership functions of input and output parameters [38].

\subsection{3-the fuzzy inference engine}

is considered the central part of the fuzzy system it contains the Knowledge Base, that stores IFTHEN rules provided by experts. It simulates the human reasoning process by making fuzzy inference on the inputs and IF-THEN rules, combines rules from the fuzzy rule base and gives a mapping from input fuzzy sets, each rule is interpreted as a fuzzy implication. In this step the inference engine is applied to a set of rules included in the fuzzy rule base and that to produce the intended output. This procedure involves many steps as follows: first, it matches the linguistic variables of the input with the rules' premises. Second, it activates the matched rules in order to deduce the resultant of each fired rule, and finally it combines all consequents by using fuzzy set union in order to generate the final output which is represented as fuzzy set output [37]. 


\subsection{4- the Defuzzifier}

It transforms the fuzzy set obtained by the inference engine into a crisp value, the output is produced as a linguistic variable, which is fuzzy and can be interpreted in different ways. The process of finding a crisp output after fuzzification and inference is called Defuzzification Therefore, the fuzzy set output in this stage is converted to a crisp output (which results on a fuzzy output set).

\subsection{Advantages of Using Fuzzy Logic with QA System}

QA Question answering system enable users to access the knowledge in a natural way by asking questions and get back relevant correct answers, and that requires understanding of natural language text, linguistics and common knowledge, derived from the documents and providing accurate answers to a user's question often expressed as uncertainty words. So many of previous researchers on how to build QA logics, find that using artificial intelligence (AI) based methods that integrate natural language processing (NLP) techniques and knowledge base or corpus help to build QA logics. And they found that using fuzzy logic to modify linguistic values can retrieve the answers by matching user's question with the existing hierarchical ontology, and not only provide syntactic answers, but also semantic answers based on the question terms. It can produce result sets according to the degree of vague expressions in natural language, and vague adjectives in particular. And also, one of the main strengths of Fuzzy logic is that it allows the semantic partitions to overlap. In (1988) Smithson (argues that psychological explanations that permit choice under partial and uncertain constraints can be compatible with fuzzy logic [39]. For that Fuzzy logic is widely used in a different field such as expert systems, business and medicine. It can measure the output of the process and takes control actions on the process continuously.

Using fuzzy logic in NLP make the possibility of constructing a theory for artificial languages whose terms have fuzzy meaning and contribute it to a clarification of the concept of semantic meaning. Fuzzy logic, has a direct relation to NLP and QA development, A Critical Survey on the use of Fuzzy in Natural Language Processing start from ( 2004, Araujo) for rule learning, in 2010 was for knowledge representation by and in 2011 by Lai, Wu, Lin, \& Huang,), research was for word meaning inference in (2012,Carvalho,Batista,\&Coheur)for linguistic summarization[38]. in 2013was two search the first one was about grammatical inference ,by( (Kazemzadeh, Lee, \& Narayanan)and the second one was for emotion recognition by (Luong, Socher, \& Manning) in 2014 by (Cambria, Gastaldo, Bisio, \&Zunino) for text categorization . most of the researchers currently working in the NLP field with fuzzy logic in different aspects, tries to find some guidelines on what could be done to and make fuzzy logic more interfere with NLP. Therefore, many of the previous researchers relied on used fuzzy logic in NLP have many advantage as it can represent the meaning of dispositions provide with more accuracy in expressive with easy-to-use operators provides simpler framework that lead to increase the accuracy of recognition system efficient. and also, fuzzy logic can use as translators or framework to match scores higher than before across different text domains.

\section{CONCLUSIONS}

in this survey paper are show an overview on what Question-Answering is and its architecture and the possible relationship with fuzzy logic and how fuzzy logic can be used as intermediate semantic representations of vague expressions. we pin down the previous related research with summarized and organized recent research results in a novel way that integrated and added 
understanding to work in the question-answering field. It emphasized the classification of the existing literature, developing a perspective on the area, and evaluating trends. However, because it is impossible for a survey to include all or even most of previous research, this survey included only the work of the top-publishing and top-cited authors in the QA field.

\section{FUTURE WORK}

In this paper it has been discussed some of the elementary approach, and it perform fairly well but suffer from some limitations. This fact leads us to next step as a next step, we will try to tame our feature seton one possibility is to development a QA system using hybrid approach in our system that will be designed to be able to answer number of questions mainly based on fuzzy rule that is less impacted by the serious imbalance between negative and positive instances. This should help to increase the accuracy of Future result the architecture of Future Question Answering system using fuzzy logic rule is as shown in Figure 3.

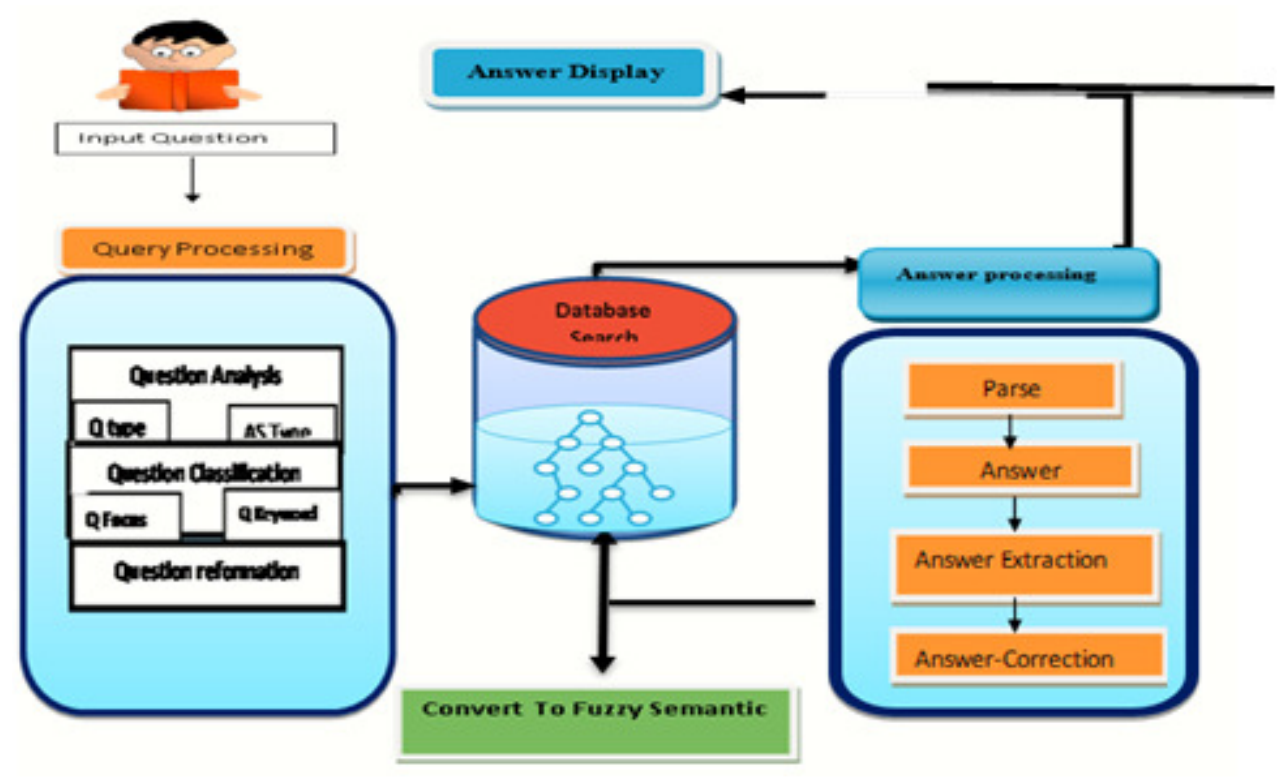

Figure 3. Question Answering System Using Fuzzy Logic Rule

\section{REFERENCES}

[1] Hendrix. G.G, Sacerdoti, E.D,sagalowicz. D. Slocum. J. "'Developing a natural Language interface tocomplex data in ACM Transaction on database system. 3(2). pp. 105- 147,1978.

[2] Green BF, Wolf AK, Chomsky C, and Laughery K. Baseball: An automatic question answerer. In Proceedings of Western omputing Conference, Vol. 19, 1961, pp. 219-224.

[3] [Cook, 2006] Cook, J. L. (2006). College students and algebra story problems: Strategies for identifying relevant information. Reading Psychology, 27:95 - 125.

[4] Weizenbaum J. ELIZA - a computer program for the study of natural language communication between man and machine. In Communications of the ACM, Vol. 9(1), 1966, pp. 36-45 
[5] Woods W. Progress in Natural Language Understanding - An Application to Lunar Geology. In Proceedings of AFIPS Conference, Vol. 42, 1973, pp. 441-450.

[6] Bobrow, D. G., Kaplan, R. M., Kay, M., Norman, D. A., Thompson, H., and Winograd, T. (1977). GUS, A frame driven dialog system. Artificial Intelligence, 8, 155-173.

[7] Julian Kupiec. 1993. MURAX: A robust linguistic approach for question answering using an on-line encyclopedia. In Robert Korfage, Edie Rasmussen, and Peter Willett, editors, Proceedings of the Sixteenth Annual International ACM SIGIR Conference on Research and Development in Information Retrieval, pages 181-190. Special issue of the SIGIR FORUM

[8] Lehnert, W. G. (1978). The Process of Question Answering: A Computer Simulation of Cognition. Hillsdale, NJ: Lawrence Erlbaum Associates, Inc.

[9] Huettner, A. (2000) "Question Answering”. In: 5th Search Engine Meeting.

[10] Voorhees EM. The TREC-8 question answering track report. In Proceedings of TREC-8, 1999, pp. 77-82.

[11] K.L.Kwok,L.Grunfeld,N.Dinstl,and M.Chan.TREC-9 Cross language,web and quationt -answering track experiments using PIRCS.In Voorhees and Harman .

[12] E.M.Voorhees and D.K. Harman,editors,Proceedings of the Ninth Text REtreival Conference (TREC9)

[13] Stefanie Tellex, "Pauchok: A Modular Framework for question Answering", Master Thesis Submitted to the Department of Electrical Engineering and computer science, Maccachusetts institute of Technology, June 2003.

[14] Michael Kaisser. Question Answering by Searching Large Corpora with Linguistic Methods. Master's thesis, Saarland University, Germany, 2004.

[15] Riloff E and Thelen M. A Rule-based Question Answering System for Reading Comprehension Tests. In ANLP /NAACL Workshop on Reading Comprehension Tests as.

[16] Ittycheriah A, Franz M, Zhu WJ, Ratnaparkhi A and Mammone RJ. IBM's statistical question answering system. In Proceedings of the Text Retrieval Conference TREC-9, 2000.

[17] Joho, H. (1999) "Automatic detection of descriptive phrases for Question Answering Systems: A simple pattern matching approach". MSc Dissertation. Department of Information Studies, Universityof Sheffield. Sheffield, UK

[18] Deepak Ravichandran, Abraham Ittycheriah and salimroukos, "Automatic Derivation of surface text pattern for a maximum Entropy Based question answering system", Work done while the author was an intern at IBM TJ Watson research center during summer 2002.

[19] Cai D, Dong Y, Lv D, Zhang G, Miao X. A Web-based Chinese question answering with answer validation. In Proceedings of IEEE International Conference on Natural Language Processing and Knowledge Engineering, pp. 499-502, 2005.

[20] J.-L. Binot, L. Debille, D. Sedlock, and B. Vandecapelle. Natural Language Interfaces: A New Philosophy. SunExpert Magazine, pages 67\{73, January 1991 
[21] Berger A, Caruana R, Cohn D, Freitag D, and Mittal V. Bridging the lexical chasm: statistical approaches to answer-finding. In Proceedings of the 23rd annual international ACM SIGIR conference on Research and development in information retrieval, 2000, pp. 192-199.

[22] Bronnenberg, W.J., Bunt, H.C., Landsbergen, S.P.J., Scha, RoJ.H., Schoenmakers, W.J., van Utter,n, E.P.C. (1979) The question answering system PHLIQAI. In L.Bolc (ed.), Natural communication with computers, McMillan, London; Hanser Verlag, M nehen.

[23] SACERDOTI, E.D. Language access to distributed data with error recovery. Proc. 5th Int. Joint Conf. on Artificial Intelligence, Cambridge, Mass., Aug. 1977.

[24] Ask Jeeves. 1996. www.ask.com Site last visited in 28-march-2018.

[25] AnswerBus, Question Answering System. Website: http://answerbus.com J. Allen. 1995. Natural Language Understanding. The Benjamin/Cummings Publishing Company, Menlo Park, CA.

[26] Vargas-Vera M. and Motta E and Domingue J. (2003a): AQUA: An Ontology-Driven Question Answering System. AAAI Spring Symposium, New Directions in Question Answering, Stanford University, March 24-26, 2003.

[27] Boris Katz, Sue Felshin, Deniz Yuret, Ali Ibrahim, Jimmy Lin, Gregory Marton, Alton Jerome McFarland, and BarisTemelkuran. 2015. Omnibase: Uniform access to heterogeneous data for question answering. In Proceedings of the 7th International Workshop on Applications of Natural Language to Information Systems (NLDB 2015).

[28] Unmeshsasikumar, Sindhu L, "A survey of Natural Language question answering system", international journal of computer applications(0975-8887), volume 108 -No 15. December 2014.

[29] Anthony Fader, Luke Zettlemoyer, and Oren Etzioni. 2014. Open question answering over curated and extracted knowledge bases. In Proceedings of the 20th ACM SIGKDD International Conference on Knowledge Discovery and Data Mining, KDD '14, pages 1156-1165, New York, NY, USA. ACM

[30] Sanjay K. Dwivedi and vaishalisingh "Research and reviews in question answering system", International Conference on Computational Intelligence: Modeling Techniques and Applications CIMTA) 2013 Procedia Technology 10 ( 2013 ) 417 - 424.

[31] Ellen M. Voorhees and Dawn M. Tice. 1999. The TREC-8 question answering track evaluation. TREC-8 Proceedings, pages 41-63, Nov.

[32] JIPING SUN, FAKHRI KARRAY, Fuzzy Logic-Based Natural Language Processing and Its Application to Speech Recognition, Ontario, N2L 3G1, Canada,2017

[33] J.Berant, A. Chou, R. Frostig, and P. Liang, "Semantic parsing on freebase from question-answer pairs," in Proceedings of the Conference on Empirical Methods in Natural Language Processing (EMNLP '13), vol. 2, p. 6, 2013. View

[34] F. J. Estrella, M. Espinilla, F. Herrera, and L. Martínez, "FLINTSTONES: a fuzzy linguistic decision tools enhancement suite based on the 2-tuple linguistic model and extensions," Information Sciences, vol. 280 , pp. 152-170, 2014

[35] V. C. Gerogiannis, E. Rapti, A. Karageorgos, and P. Fitsilis, "A fuzzy linguistic approach for human resource evaluation and selection in software projects," in Proceedings of the 5th International Conference on Industrial Engineering and Operations Management (IEOM' 15), pp. 1-9, Dubai, UAE, March 2015. 
[36] VAN RIJSBERGEN, C.J. Information Retrieval, Second Edition, Butterworths, London (1979).

[37] L. A. Zadeh, "A computational approach to fuzzy quantifiers in natural languages", Comput. Math. Appl., vol. 9, pp. 149-184, 1983.

[38] A. Niewiadomski, "A type-2 fuzzy approach to linguistic summarization of data", IEEE Trans. Fuzzy Syst., vol. 16, no. 1, pp. 198-212, Feb. 2008.

[39] VilémNovák,Fuzzy Systems (FUZZ-IEEE), 2017 IEEE International Conference on, 10.1109/FUZZIEEE.2017.8015405, Naples, Italy, 8, 2017

[40] Zadeh, L. A. (1978) ,PRUF-A Meaning Representation Language for Natural Languages, Int. Journal Man-Manchine Studies, 10, pp. 395-460; also in Fuzzy Sets and Applications: Selected Papers by L. A. Zadeh, John Wiley \& Sons, New York, pp. 499-568 (1987).

[41] Konstantinos Chatzitheodorou, Proceedings of the Workshop on Natural Language Processing (NLP4TM)Improving translation memory fuzzy matching by paraphrasing, pages 24-30, Hissar, Bulgaria, Sept 2015

[42] L. Zadeh. Test-Score Semantics as a Basis for a Computational Approach to the Representation of Meaning. Literary and Linguistic Computing, 1986.

[43] Fenza, G., V. Loia, and S. Senatore. 2008. "Concept Mining of Semantic Web Services by Means of Extended Fuzzy Formal Concept Analysis (FFCA)." IEEE Int. Conf. on Systems, Man and Cybernetics

[44] Monika Rani, Maybin K. Muyeba, O.P. Vyas, A hybrid approach using ontology similarity and fuzzy logic for semantic question answering." In Advanced Computing, Networking and Informatics Volume 1, pp. 601-609. Springer, Cham, 2014.

\section{AUTHOR}

Eman Mohamed Nabil Alkholy

Faculty of Computers \& Information, Helwan University, Cairo, Egypt.

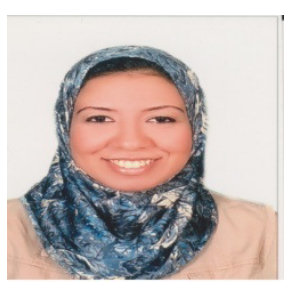

